\title{
LÉGISLATION
}

\section{Les Sociétés Coopératives et les Groupements Coopératifs en présence des Lois fiscales de toutes catégories (résumé)}

\author{
par Paul Bougault, avocat à la Cour d'appel de Lyon.
}

\begin{abstract}
Nole de la Rédaclion. - - Nous sommes heureux d'annoncer à nos lecteurs que la Librairic des Editions J. Rey-B. Arthaud, successcur, à (irenoble, vient de mettre en vente le Manuel fiscal des Sociétés Coopéralives et Groupements coopératifs, au prix de 16 francs, de notre collaborateur Paul Bougault, avocat à la Cour d'Appel de Lyon; manuel qui reproduit avec de multiples développements les articles qui ont paru dans La Houille Blanche pendant le cours de l'année 1926. On trouvera ci-dessous l'exposé des dernières discussions qui, sans faire avancer la question, ont eu lieu au Parlement.
\end{abstract}

Situation stationnaire des travaux parlementaires entre le 13 juillet 1925 et le 30 juin 1926. - Dans notre dernier article (voir le numéro de juillet-août 1926), nous avons indiqué en les résumant, quelles élaient les tentatives de réforme faites au Parlement pour le chiffre d'affaires des Sociétés Coopératives.

Il nous reste à rechercher comment a été utilisé par le Parlement et le Gouvernement ce délai d'un an environ, allant du 13 juillet 1925 an 30 juin 1926 .

On sait, en effet, qu'à cette dernière date, a paru une loi dont l'arlicle 21, bien qu'il ne fut relatif qu'aux Syndicats agricoles, a créé une certaine émotion.

Nous pouvons dire que jusqu'à cette dernière date, nous avons assisté à un chassé-croisé de projets déposés et de renvois à la Commission qui s'est produit dans les circonstances suivantes :

Le 19 décembre 1925 , le député Boully dépose une proposition de loi (No 2279), ayant pour but d'instaurer pour toutes les Coopératives, un système d'imposition dégrevant celles qui sont plus particulièrement chargées avec les tarifs actuels.

On peut considérer, comme une réponse à cette proposition un article supplémentaire $2 \mathrm{M}$ qui, inséré par la Commission des Finances dans son rapport supplémentaire No 2743 sur le projet de budgel de 1926, proposait, avec une certaine soudaineté, à la Chambre, de se borner en définitive, à l'exonération des Syndicats agricoles, purement et simplement, en s'abstenant de toute incursion dans le domaine des Coopératives.

La Chambre des Députés mise en présence des deux systèmes renvoya l'article $2 \mathrm{M}$ à la Commission (voir la scconde séance du 23 mars 1926).

Situation modifiée en juin 1926 pour les Syndicats agricoles seulement. - Loi du 30 juin 1926, article 21. -- Les choses en étaient encore à ce point, au début de l'été 1926, lorsque le Gouvernement fut obligé, comme chaque année, de saisir le Parlement d'un projet de loi ayant pour effet d'autoriser et de régulariser, par des crédits supplémentaires, les dépenses afférentes aux charges publiques rendues obligatoires par la loi de Finances, dont le montant ne peut être reconnu définitivement qu'après l'exécution des services. C'est une loi vraiment budgétaire, dans laquelle on ne devait traiter que des question de chiffres. Mais le
Gouvernement profita de l'occasion pour greffer, sur son projet de crédits supplémentaires, un article 19 qui représente à peu près l'ancien article supplémentaire du projet du budget de 1926, No 2743, et par conséquent, ne concerne que les Syndicats agricoles.

La Commission reçut de la Chambre l'ordre formel d'examiner à nouveau la prétention du Gouvernement, et une Sous-Commission composée de MM. de Monicault, Prevet et Locquin fit alors un travail approfondi, présentant à la Chambre, au lieu d'un seul article 19, trois articles portant les Nos 21, 22 et 23.

L'article 21 du projet exempte les affaires des Syndicats agricoles, en rappelant que cette exemption n'est acquise évidemment qu'à ceux qui sont dispensés de l'impôt sur les bénéfices industriels et commerciaux (ventes aux seuls sociétaires, el ristourne des bonis sans bénéfice), à la condition que les affaires portenl bien sur des produits ou objets nécessaires à l'exercice de la profession d'agriculteur (cet article a été adopté par la Chambre à la séance du 30 juin 1926, Journal officiel du $1^{\mathrm{er}}$ juillet, page 2643).

L'article 22 du projet considère les Sociétés coopératives de consommation comme des mandataires, à la condition que ces Sociétés ne vendent qu'à leurs seuls sociétaires, ne rémunèrent que leurs employés et encore seulement par un traitement fixe, et enfin ristournent la totalité de leurs trop perçus à leurs seuls sociétaires au prorata de la consommation de chacun, ou à des cuvres d'intérêt général : le chiffre d'affaires ne sera alors perçu que sur le montant des bonis affectés au paiement des frais généraux et à la constitution des réserves. (Cet article a été adopté par la Chambre à la séance du 30 juin 1926. Journal officiel du

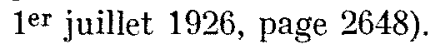

L'article 23 considère comme mandataires les Groupements d'achats en commun, avec ou sans stock, en vue de l'achat et de l'attribution à leurs seuls membres, des marchandises nécessaires à leur production. Dans ce cas, la taxe sur le chiffre d'affaires n'est perçue que sur le montant des frais généraux ou des commissions (cet article a été adopté à la séance du 30 juin 1923 ,

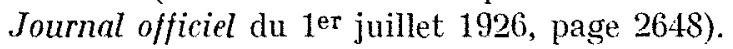

C'est en cet état que le projet de loi arriva au Sénat le 30 juin 1926 au soir, vers 19 heures, c'est-à-dire an milien de celle séance qui devait se terminer le $1^{\text {er }}$ juillet 1926 , à 7 heures du matin.

Le rapporteur au Sénat, M. Chéron, fit son rapport en séance même (voir Sénat, Débats parlementaires, $2^{e}$ séance du 30 juin 1926, Journal officiel du $1^{\text {er }}$ juillet, page 1275). Il proposa, au nom de la Commission, de supprimer tout ce qui regardait les Coopératives et de cantonner l'exemption du chiffre d'affaires dans le domaine des seuls Syndicats agricoles pour les motifs suivants : "Les Syndicats agricoles, en général, achètent sur 
" commandes préalables, c'est-ì-dire pour répartir et non pour "revendre; ils se trouvent ainsi légalement exonérés de la laxe a sur le chiffres d'affaires. Quelquefois, cependant, ils ne peuvent " justifier des commandes préalables de leurs adhérents et alors, " en vertu de la jurisprudence du Conseil d'Etat, ils seraient " soumis à la taxe sur le chiffre d'allaires. Mais ce sont là des cas " exceptionnels. Ils ne sauraient justifier, pour la règle géné" rale, l'assujettissement à la taxe sur le chiffre d'affaires.

" Les Coopératives de consommation, de leur côté, sont, de " toute évidence, des organismes très intéressants. Mais étant " donné surtout qu'elles reçoivent déjà des avantages de l'Etat, " et qu'elles sont ainsi mises en mesure de faire bénéficier leurs " adhérents de prix avantageux, il ne nous paraît pas possible, " quand elles font acte de commerce, de les exonérer de charges "que supportent les commerçants auxquels elles font concur"rence.

"Quant aux Groupements d'achats en commun entre profes" sionnels, ils ont toujours été considérés comme imposables à la ( taxe sur le chiffres d'affaires. Ils tendent à l'heure actuelle à se " substituer au commerce de gros et, s'ils bénéficiaient désormais " d'une exonération à la taxe sur le chiffre d'affaires, il en résul" terait, pour le Trésor, une perte de recettes appréciable. Pour " ces motifs, nous vous proposons, comme le Gouvernement, de " limiter aux seuls Syndicats agricoles et Unions de Syndicats, le " bénéfice de l'exonération de la taxe sur le chiffre d'affaires. Tou" tefois il lui a semblé qu'il convenait de retenir la restriction " demandée par notre Assemblée et elle a modifié dans ce sens le " texte primitif."

En conséquence, le rapporteur donnait le texte unique qui devait remplacer les trois articles adoptés par la Chambre.

Il était évident que, à cette heure tardive, la loi devant être promulguée le lendemain matin, la discussion au Sénat ne pouvait être bien longue. Elle fut néanmoins assez animée, comme on peut le voir au Journal officiel déjà cité du 1er juillet 1926 (Débats parlementaires, Sénat, pages 1285 et suivantes) et l'article 21 fut voté (voir ibidem, page 1290 in medias).

Voici le texte définitif : "Sont exemptées de l'impôt sur le " chiffre d'affaires, les affaires effectuées par les Syndicats agri" coles réunissant les conditions requises pour bénéficier de l'exo" nération de l'impôt sur les bénéfices industriels et commer" ciaux, par application des dispositions de l'article 15 de la loi " du 31 juillet 1917, ainsi que celles effectuées par les Unions " constituées entre lesdits Syndicats, à condition que lesdites " affaires ne portent que sur les produits ou objets nécessaires " à l'exercice de la profession d'agriculteur."

Nouvelle discussion sans résultat reprise pour les Coopératives en 1927. - A la séance du Sénat du 18 mars.1927, (Journal officiel du 19 mars 1927, Débats parlementaires, Sénat, page 285 et suivantes) la discussion fut reprise. A ce moment le Sénat s'occupait de la loi budgétaire destinée à régulariser les crédits ouverts par des décrets au titre de l'exercice 1926 et de l'ouverture et de l'annulation de crédits sur l'exercice 1926 au titre du budget général et des budgets annexes. Le Sénat avait profité de cetle occasion pour statuer sur l'exemption de la taxe accordée aux établissements d'enseignement, lorsque MM. Louis Soulié, Fernand Merlin, Drivet et Delay présentèrent un amendement ainsi conçu :

"Sont considérées comme des opérations de mandat, et taxées " sur le montant des bonis bruts prélevés sur ces opérations, soit " pour le paiement des frais généraux, soit pour la constitution des " réserves : "a) Les affaires trailices avec leurs membres par les Groupe"ments d'achats en commun, sans slocks ou avec stocks, cons" titués exclusivement entre professionnels, persomes ou Sociétés " en vue de l'achat de la vente à leurs seuls membres de marchan"dises ou objets nécessaires à l'exercice de leur profession. "

Cet amendement était la généralisation de la laxe sur le boni (sur lo boni seulement et non pas sur le prix de la vente) même pour les Sociétés coopératives de consommation pratiquant l'achat pour la revente, avec stock eu sans stock. Une nouvelle disjonction a été votée après une discussion dans laquelle on a paru étrangement confondre les cooperatives vraies ot les fausses Coopératives. Mais la disjonction a élé basée sur une question de discipline à observer dans les voles du Parlement el qui a élé rappelée en ses termes par M. Poincaré, ministre des linances : "Je " ne veux par reprendre une discussion qui s'étend des Coopéra" tives de consommation aux Coopératives de produclion et " qui finirait certainement dans la confusion. Il ne s'agil pas " de refaire aujourd'hui des lois qui sont votées. La discussion " du mois de juin 1926 a été longue et approfondie et, si je ne " me trompe pas, tous les arguments, à ce moment, ont été " fournis pour et contre. C'est donc en pleine connaissance de " cause que le Sénat a considéré, comme portant atteinte au " principe de l'égalité devant l'impôt, la disposition que reprend " aujourd'hui l'honorable M. Soulié. Mais j'ajoute que cet " amendement me paraît se greffer sur le projet de loi en dis" cussion dans des conditions tout a fait anormales. Je prends " le texte mème de l'amendement et je vous demande la permis" sion de le relire. Je vous assure que c'est là qu'apparaît le " paradoxe dont parlait tout à l'heure l'honorable M. Merlin : "Amendement au projet de loi adopté par la Chambre des dépu" tès portant : $1^{\circ}$ Régularisation de crédits ouverts par décrets " au titre de l'exercice de 1926 ; $2^{\circ}$ ouverture et anmulation de " crédits ouverts par décrets au titre de l'exercice de 1926; " $2^{\circ}$ Ouverture et annulation de crédits sur l'exercice de 1926 " au titre du budget général et des budgets annexes. Voilà bien " circonscrit l'objet du débat actuel : il s'agit de crédits à ouvrir" " ou à annuler sur l'exercice 1926. Or, que nous proposc-t-on ? "Article additionnel 64 bis : Après l'article 64, insérer un article " additionnel 64 bis ainsi conçu : Sont considérées comme des " opérations de mandat et taxées sur le montant des bonis bruts " prélevés sur ces opérations, etc. Laissez-moi vous dire que je " me crois à la Chambre des dépulés avant la période de sages e " que j'ai connue depuis six mois. En réalité, nous rentrons dans "le désordre budgétaire. Nous sommes en présence d'un texte " précis et nous y ajoutons des greffes tout à fait parasites. Je. " supplie le Sénat d'écarter, pour raison de méthodes, l'amende"ment proposé. "

Sur cette observation du Président du Conseil, l'amendement a èté repoussé.

Etat actuel résultant de toutes les dispositions législatives et de la jurisprudence. - Réponses données aux parlementaires par le Ministère des Finances. - Il semble, au premier abord, bien simple de résumer l'état acluel de la question du chiffre d'affaires en ce qui concerne les Syndicals, Sociétés coopératives, Groupements d'achat en commun, pusque l'on ne doil, en résumé, consulter que deux lois : l'article 85 de la loi du 13 juillet 1925 et l'article 21 de la loi du 30 juin 1926 ; mais le nombre des personnes qui consentent à lire des textes avant d'en parler est assez restreint, et c'est pourquoi on entend exprimer lant d'opinions divergentes.

La situation est la suivante, au moment où sont imprimées ces lignes (fin octobre 1927). 
$1^{\circ}$ En ce qui concerne les Syndicats agricoles, la question est réglée par l'arlicle 21 de la loi du 30 juin 1926. L'exemption est absolue, même quand un Syndicat agricole " fait la vente à ses "adhérents ", à la condition toutefois qu'il remplisse les conditions pour être exonéré de l'impôt sur les bénéfices industriels et commerciaux (voir ci-dessus chapitre III), la vente ne devant porter que sur un produit ou un objet nécessaire à la profession d'agriculteur ;

$2^{\circ}$ En ce qui concerne Loutes les Coopératives, et tous les Groupements d'achat en commun, la question est également réglée puisqu'il sufflt d'appliquer l'article 85 de la loi du 13 juillet 1925 ; même les firme; dispensées de l'impôt sur les bénéfices industriels el conmerciaux tombent sous le coup de la taxe sur le chiffe d'affaires, si elles pratiquent l'achat pour revendre (la revente fût-elle même faite à un des șociétaires et à plus forte raison à d'aulres personnes), car l'acte d'acheter pour revendre est un acte essentiellement commercial qui expose celui qui le commet, aussi bien à titre exceptionnel qu'à titre permanent, à l'impôt sur le chiffre d'affaires d'après les principes de l'article 59 de la loi du 25 juin 1920.

$3^{\circ}$ Enfin, quand il n'existe pas de vente, par conséquent quand il n'y a pas de prix versé pour une marchandise cédée, mais simplement une commission remise à la Société coopérative ou au Groupement d'achat en commun pour rémunérer le service rendu par cette Société ou ce Groupement, il ne saurait y avoir la moindre perception de la taxe, même sur le boni, même sur les sommes consacrées aux frais généraux. Toutefois, ce droit à l'exonération n'est acquis que si la Société coopérative ou le Groupement considéré remplit les conditions exigées par la loi du 31 juillet 1917 pour l'exemption de l'impôt sur les bénéfices industriels et commerciaux, ou, ce qui est la même chose, exigées par l'article 18 du décret de codification du 15 octobre 1926.

En effet, comme l'ont formellement déclaré les deux arrêts du Conseil d'Etat le 18 janvier 1924, si l'opération de l'achat pour la revente entraîne la perception de la taxe sur le chiffre d'affaires en vertu de la première partie de l'article 59 de la loi du 25 juin 1920 , toute autre opération, même commerciale, si clle est exemptée sur l'impôt des bénéfices industriels et commerciaux, ne saurait entraîner cette perception en vertu de la seconde partie de cet article, il suffit de le lire pour s'en rendre compte. "Il est institué " un impôt sur le chiffre des affaires faites en France par les " personnes qui, habiluellement, ou occasiomnellement, achètent " pour revendre... (c'est la première partie), ou accomplissent " des actes relevant des professions assujetties à l'impôt sur les "bénéfices industriels et commerciaux... (c'est la seconde partie)."

On voit donc que, si la question à résoudre apparaît à juste titre comme complexe, la solution qu'elle comporte n'est pas très compliquée. Aussi, il est étonnant que l'on ait été obligé d'attendre jusqu'au Journal officiel du 21 août 1927, pour obtenir du Ministère des Finances une réponse vraiment complète aux questions posées par les Députés ou Sénateurs. Il n'en est que plus intéressant de connaître celle qui va suivre : nous la reproduisons en imprimant en caractères italiques la partie qui est relative à la taxe sur le chiffre d'affaires, ct qui est rigoureusement conforme aux principes que nous venons d'exposer :

M. Grinda, député, ayant demandé le 14 juin 1927 , sous le No 12786 , à M. le Ministre des Finances, quelle est la situation, au regard de l'impòt sur les bénéfices commerciaux de la taxe sur le chiffre d'affaires des Coopératives et des Economats, a reçu cette réponse :

"Aux termes de l'article 18 des lois codifiées par le décret du
" 15 octobre 1926, les Sociétés coopératives de consommation sont " passibles de l'impôt sur les bénéfices industriels et commerciaux " quand elles possèdent des établissements, boutiques ou maga" sins pour la vente ou la livraison de denrées, produits ou mar" chandises.

"En sont toutefois affranchies les Coopératives de consomma" tion qui se bornent à grouper les commandes de leurs adhérents " et à distribuer, dans leurs magasins de dépôt, les denrées, pro" duits ou marchandises ayant fait l'objet de ces commandes, ou " lorsque, ne vendant qu'à leurs sociétaires, elles distribuent "leurs bonis aux dits sociétaires, ou à des œuvres d'intérêt géné" ral, ou lorsqu'elles consacrent ces bonis à des réserves qui ne " sont pas réparties entre les porteurs d'actions. Quant aux Econo" mats annexés à certaines entreprises, ile ne se trouvent pas, " en principe, dans le cas d'être assujettis à l'impôt en question, " attendu que l'article 3 de la loi du 25 mars 1910 (article 77 du " titre premier du Code du Travail) qui règle le fonctionnement " de ces organismes, stipule expressément que leur existence ne " peut être tolérée que tout autant qu'ils ne sont pas productifs " de bénéfices."

"En ce qui concerne la taxe sur le chiffre d'affaires, les Coo" pératives de consommation et les Economats en sont passibles dans "les conditions de droit commun, c'est-à-dire sur le montant de "leurs ventes lorsqu'ils achètent pour revendre, et sur le montant " de leur rémunération lorsqu'ils se bornent à grouper des com" mandes et à répartir ensuite entre les ayant-droits des marchan" dises achetées en vertu de ces commandes."

"Toutefois, pour les opérations de cette dernière catégorie, les "Coopératives de consommation sont totalement exemptées de ladite " taxe lorsque, remplissant les conditions requises pour être exo" nérées de l'impôt sur les bénéfices industriels et commerciaux en " vertu des dispositions requises sous l'article 18 du décret de codi" fication du 15 octobre 1926, elles se trouvent par là-même ne " rentrer dans aucune des catégories redevables visées par le pre" mier paragraphe de l'article premier du décret de codification du " 28 décembre 1926."

Il est facile de remarquer que cette réponse est profondément. différente de celle qui figure au Journal officiel du 3 décembre 1925 et qui, ne contenant pas la restriction indiquée dans les termes imprimés en caractères italiques ci-dessus, est évidemment erronée. Le député Paul Bellamy avait demandé le 5 novembre 1925 , sous le No 5506 , si l'article 85 de la loi du 13 juillet 1925 soumettait à l'impôt sur le chiffre d'affaires tous les Groupements d'achats en commun constitués entre professionnels, quelles que soient leur forme et la nature de leurs opérations; et il précisait expressément qu'il envisageait le cas d'un Groupement d'achats en commun constitué entre professionnels dont le gérant choisi parmi les adhérents se borne à n'effectuer les achats que sur commandes préalables des membres désignant la quantité et la nature des marchandises, à les faire expédier directement par les vendeurs aux acheteurs, à prélever une commission de $0.50 \%$ pour payer les frais généraux et à répartir les bonis en fin d'année aux membres adhérents au prorata de leurs achats et, dans laffirmative, si le chiffre d'affaires est constitué par le montant des bonis réalisés grâce au groupement, ou par le montant des ordres d'achat transmis par le gérant. Ce député a reçu la réponse suivante qui est inconciliable avec les principes et avec la réponse figurant au Journal officiel du 21 août 1927. "Le "Groupement d'achat en commun envisagé sera soumis à la " taxe sur le chiffre d'affaires à compter du 1er janvier 1926. "Mais étant données les conditions dans lesquelles il fonctionne, " la taxe ne sera exigible que sur le montant des commissions, 
" sous déduction des ristommes versées en lin d'année aux adhé"rents. "

Il tombe sous le sens que, si les bonis sont répartis entre les acheteurs et ne profitent en aucune façon aux porteurs de parts, limpòt sur les bénéfices industriels et commerciaux ne sera pas exigible el, par consequent, la taxe sur le chiffe daffaires ne sera pas clue.

La circulaire du 22 juillet 1926 est également très incomplète sur le point qui nous occupe. Elle ne vise que les Groupements dachats en commun qui, agissant comme commissionnaires, trouveraient une source de bénéfice dans les commissions. Elle oublie complètement les Groupements d'achats en commun qui agissent comme de véritables Coopératives, el par consẻquent sont soustraits à l’impôt sur les bénéfices industriels et commerciaux et, par voie de conséquence, à la taxe sur le chiffred'affaires. Il est facile de se rendre compte de cette insuffisance en lisant le libellé de la circulaire précitée : "Les Groupements qui, " à la différence des Syndicats agricoles et des Coopératives de " consommation sont, dans tous les cas, tributaires de l'impòt " sur les bénélices industriels et commerciaux, et au nombre " desquels rentrent, avec les Unions des magasins de gros, les "Coopératives de consommation, les Sociétés constituées entre " les industriels et commerçants pour procurer à leurs membres " les produits ou marchandises nécessaires, sont taxés sur le " montant de leur rémunération s'ils agissent en vertu de com" missions préalables, ou sur le montant de leurs livraisons, "s'ils constituent des stocks et achètent pour revendre."

La réponse faite au député Grinda comble très heureusement cette lacune.

Observations sur certaines réponses du Ministère. - Il faut reconnaître que certaines autres réponses du Ministère des Finances, tout en étant sévères sur l'application des principes, sont inattaquables parce qu'elles sont une exacte application de la loi.

On peut citer notamment :

$1^{\circ}$ La réponse figurant au Journal officiel du 12 janvier 1927. M. Renaud, député, a exposé que, dans certaines régions, l'Administration réclame l'impôt sur le chiffre d'affaires pour toutes les marchandises livrées par les Symdicats à leurs adhérents, sous prétexte que ces Syndicats livrent des marehandises qui ne: sont pas de destinations spécialement agricoles cl demande:

$1^{\circ}$ S'il s'agit dans ce cas d'une application exacte de la législation en vigueur; $2^{\circ}$ Dans la négative, comment s'établit la distinction entre les marchandises sommises it la taxe el celles gut sont exonérées. La réponse est la suivanle : "L'article 21 de la loi " du 30 juin 1926 n'exonère les Syndicats agricoles de la laxe " sur le chiffre d'affaires que si, tout en étant affranchis de l'im" pôt sur les bénéfices industriels et commerciaux par applica" tion des dispositions de l'article 15 de la loi du 31 juillet 1917, “ils ne se livrent qu’à des opérations portant sur les produits " ou objets nécessaires à l'exercice de la profession d'agriculteur. "Par suite, les Syndicals agricoles vendant à leurs membres " l'autres produits ou objets que ceux visés, restent sous lo régime "de droits communs et sont redevables de la taxe sur le chiffre " d'affaires à raison de l'ensemble de leurs opérations. "

$2^{\circ}$ La réponse figurant au Journal officich du 24 novembre 1!126, le député Boully a exposé sous le No 2518 : qu'un groupement d'achats sollicite périodiquement de ses adhérents un ordre de prévision pour leurs besoins trimestriels, semestriels, on annuels, suivant la nature des produits et l'époque de la labrication; que, lorsqu'il possède l'ensemble de ces ordres, le Directeur du Groupement fait ses achats en conséquence et les factures, au fur et à mesure des besoins de ses adhérents, el demande si, les factures établies sont passibles de l'impôt sur le chiffre d'alfaires, sur leur montant brut ou sur la commission percue (question du 12 novembre 1926). La réponse est la suivante : de simples bulletins de prévisions ne sauraient être constitués comme des ordres d'achats préalables; celte dernière expression ne peut, en effet, s'entendre que d'ordres engageant ceux qui les donnent ì prendre livraison de la marchandise et réduisant l'intermédiaire qui les exécute au rôle d'un simple mandataire n'ayan que la possibilité de facturer cette même marchandise au prix d'achat augmenté d'une commission déterminée. Dans ces conditions et comme, par ailleurs, il ressort des termes de la question que le Groupement envisagé possède un magasin de ventes et constitue des stocks, ce Groupement ne peut qu'être répulé acheter jour revendre et doit, en conséquence, la taxe sur le chiffre d'affaires sur le montant intègral de ses livraisons. 